\title{
QT interval prolongation during spontaneous episodes of hypoglycaemia in type 1 diabetes: the impact of heart rate correction
}

\author{
T. F. Christensen $\cdot$ L. Tarnow $\cdot$ J. Randløv . \\ L. E. Kristensen • J. J. Struijk • E. Eldrup • \\ O. K. Hejlesen
}

Received: 12 January 2010 / Accepted: 30 April 2010/Published online: 23 May 2010

(C) Springer-Verlag 2010

\begin{abstract}
Aims/hypothesis Prolongation of the heart rate corrected QT interval $(\mathrm{QTc})$ is seen during episodes of hypoglycaemia in type 1 diabetes. We studied the relationship between spontaneous hypoglycaemia and the QT interval and hypothesised that the choice of heart rate correction affects the observed change in QTc.

Methods Twenty-one participants with type 1 diabetes (aged $58 \pm 10$ years with duration of diabetes $34 \pm 12$ years) had continuous glucose and ECG monitoring for $72 \mathrm{~h}$. QT and $\mathrm{RR}$ intervals were measured during hypoglycaemia (blood glucose or continuous glucose measurements $\leq 3.5 \mathrm{mmol} / \mathrm{l}$ ) and compared with euglycaemia $(5-12 \mathrm{mmol} / \mathrm{l})$. QT intervals were measured using the semi-automated tangent method from signal-averaged ECG and corrected using Bazett's formula, Fridericia's formula, the nomogram method and a linear subject-specific method.

Results Hypoglycaemia was present in 14 participants. With Bazett's formula, QTc changed significantly from euglycaemia to hypoglycaemia ( $422 \pm 30$ vs $432 \pm 33 \mathrm{~ms} ; p=0.02$ ). Heart rate, QT intervals and QTc corrected with formulas other than Bazett's were not associated with a significant change ( $p=$ $0.07-0.29$ ). During hypoglycaemia, significantly lower values
\end{abstract}

T. F. Christensen · J. J. Struijk · O. K. Hejlesen

Department of Medical Informatics, Aalborg University,

Aalborg, Denmark

T. F. Christensen $(\square) \cdot$ J. Randløv $\cdot$ L. E. Kristensen

Novo Nordisk A/S,

Brennum Park 20A1.105,

Hillerød DK-3400, Denmark

e-mail: tokefc@msn.com

L. Tarnow $\cdot$ E. Eldrup

Steno Diabetes Center,

Gentofte, Denmark of QTc compared with the subject-specific method were seen for Fridericia's formula $(p=0.02)$ and the nomogram method $(p=0.04)$.

Conclusions/interpretation Spontaneous hypoglycaemia was associated with a modest increase in QTc. Bazett's formula resulted in overcorrection of QTc while both Fridericia's formula and the nomogram method undercorrected the QTc compared with the subject-specific method during hypoglycaemia. The results may indicate that the use of a fixed heart rate correction formula can lead to misleading results in investigations of spontaneous hypoglycaemia.

Keywords Cardiac arrhythmia - Dead in bed syndrome . Heart rate correction · Hypoglycaemia - Type 1 diabetes
Abbreviations
CGM Continuous glucose monitoring
IG Interstitial glucose concentration
QTc QT interval corrected for heart rate
QTcB QT interval corrected for heart rate using Bazett's formula
QTcF QT interval corrected for heart rate using Fridericia's formula
QTcN QT interval corrected for heart rate using the nomogram method
QTcS QT interval corrected for heart rate using a linear subject-specific formula
$\mathrm{T}_{\text {end }} \quad$ End of the $\mathrm{T}$ wave

\section{Introduction}

In the early 1990s Tattersall and Gill reported a series of unexplained nocturnal sudden deaths of young people with 
type 1 diabetes [1]. The evidence suggested that this 'dead in bed' syndrome was caused by hypoglycaemia, since the patients had a history of severe nocturnal hypoglycaemia. The circumstances of the deaths, in which the patients were found in an undisturbed bed, led to the hypothesis that fatal cardiac arrhythmia had been triggered by hypoglycaemia [2]. Experimental and spontaneous hypoglycaemia has been shown to cause alterations in cardiac repolarisation [3-6]. Atrial fibrillation [3] and ventricular ectopic beats [4] have been reported during hypoglycaemia but the most notable change is a prolonged heart rate corrected QT interval (QTc) [4-6]. Prolongation of the QTc has been associated with an increased risk of sudden death [7, 8] and is therefore the variable of interest when investigating the proarrhythmic effect of hypoglycaemia. The QT interval is dependent on the heart rate, so that heart rate correction is necessary in order to allow comparison of QT intervals measured at different heart rates. There are various methods for heart rate correction, of which Bazett's formula [9] is the most popular. However, this method is criticised because of overcorrection at high heart rates and undercorrection at low heart rates $[10,11]$. Any fixed correction formula, like Bazett's, assumes the same QT/RR relation in all individuals. However, the QT/RR relation is highly individual, and a subject-specific correction formula is therefore preferred over a fixed correction formula [11]. The effect of spontaneous hypoglycaemia on QTc has been studied in both hospitalised [6, 12] and ambulant [4, 13] settings, but all studies use Bazett's heart rate correction. Hypoglycaemia can increase heart rate [14], and a significant change in QTc during hypoglycaemia could merely reflect overcorrection by Bazett's formula. In addition, fully automatic measurements of QT intervals from Holter monitor data without visual inspection by a human observer, as has been done in some studies $[4,13]$, might lead to significant errors [15].

In this study we used continuous glucose monitoring (CGM) and ECG monitoring in an ambulant setting of participants with type 1 diabetes to investigate whether episodes of spontaneous hypoglycaemia cause QTc prolongation. We investigated whether the choice of heart rate correction formula affects QTc prolongation during spontaneous hypoglycaemia.

\section{Methods}

Subjects We studied 21 participants (13 men and eight women) with type 1 diabetes who were prone to hypoglycaemia, defined as at least two episodes of severe hypoglycaemia within the last year. Assuming that twothirds of the participants would develop hypoglycaemia in the monitoring period, this sample size had $80 \%$ power to detect a mean increase in QTc of $15 \mathrm{~ms}$ or more during hypoglycaemia. All participants had a normal resting 12-lead ECG and did not have a history of known cardiovascular complications. No participant was on medication known to cause changes in the QT interval. The presence of autonomic neuropathy was assessed by standard cardiovascular tests [16]. Written informed consent was obtained from all participants and the study protocol was approved by the local ethics committee.

Procedures Participants were admitted to the clinical research unit on Thursday at 20:00 hours. ECG was measured with a frequency of $1,000 \mathrm{~Hz}$ and $1 \mu \mathrm{V}$ resolution from lead II using a digital Holter monitor (SpiderView Plus; ELA Medical, Montrouge, France). Simultaneously, interstitial glucose concentration (IG) was monitored using a Guardian RealTime Continuous Glucose Monitoring System (Medtronic MiniMed, Northridge, CA, USA) with the prevailing glucose level blinded. At 23:00 hours a cannula was placed into an antecubital arm vein so that blood samples could be taken at hourly intervals until 07:00 hours the next morning. Blood samples were centrifuged and stored for later measurement of plasma glucose. At 08:00 hours, participants were sent home with the monitoring equipment and were instructed to calibrate the CGM at least four times a day. Monitoring ended on Sunday at 20:00 hours, so a total of $72 \mathrm{~h}$ of continuous CGM and ECG data were available for each participant.

ECG measurements The ECG was analysed using custom analysis software developed in MatLab (Version 7.8.0.347; MathWorks, Natick, MA, USA). The ECG was analysed in segments of $1 \mathrm{~min}$. Templates representing the average PQRST complex in the segments were generated. The end of the $\mathrm{T}$ wave $\left(\mathrm{T}_{\text {end }}\right)$ in each template was determined automatically using the slope intersect method [17]. The QT interval was calculated as the time from the beginning of the $\mathrm{Q}$ wave to $\mathrm{T}_{\text {end }}$ in each template. All templates and associated fiducial points were reviewed manually on screen twice by each of two observers blinded to the corresponding blood glucose and the participants' clinical data. Templates were rejected if artefacts precluded reliable measurements of the QT interval. Rejected templates constituted $4 \%$ of all templates. No adjustments to the QT interval were made to reduce the subjectivity of the measurements. The mean intra-observer coefficient of variation for QT intervals was 1.6\% during euglycaemia and $2 \%$ during hypoglycaemia. The inter-observer coefficient of variation was $2 \%$ during euglycaemia and $3 \%$ during hypoglycaemia.

The median RR interval in each segment was used to correct the QT interval for heart rate. QT intervals were corrected by Bazett's formula (QTcB) [9], Fridericia's 
formula (QTcF) [18] and the nomogram method (QTcN) [19]. A subject-specific linear correction formula $(\mathrm{QTcS}=$ $\mathrm{QT}+\alpha \times[1-\mathrm{RR}])$ was also used as it has been shown to be the optimum model in most individuals [11]. Each participant-specific coefficient, $\alpha$, was calculated by fitting the euglycaemic QT/RR pairs from each participant to a linear regression model $(\mathrm{QT}=\beta+\alpha \times \mathrm{RR})$ using leastsquares regression.

Statistical analysis QTc was analysed at every measurement of hypoglycaemia defined as IG, overnight plasma glucose concentration or self-monitored blood glucose concentration $\leq 3.5 \mathrm{mmol} / \mathrm{l}$. Measurements of QTc during euglycaemia were taken at intervals of $30 \mathrm{~min}$ throughout the monitoring period, discarding periods when glucose values $<5 \mathrm{mmol} / 1$ or $>12 \mathrm{mmol} / \mathrm{l}$ had been measured within $1 \mathrm{~h}$. Median RR, QT and QTc durations during euglycaemia and hypoglycaemia were calculated for each participant. Changes in variables between euglycaemia and hypoglycaemia among participants were tested using a twotailed paired Student's $t$ test. Differences in QTc due to sex and neuropathy were tested using a two-tailed unpaired Student's $t$ test. Results are reported as mean $\pm \mathrm{SD}$ and a $p$ value $\leq 0.05$ was considered significant. Statistical analysis was performed using R version 2.9.1 (http://www. r-project.org/).

\section{Results}

Clinical characteristics A total of 14 participants (67\%) developed one or more episodes of hypoglycaemia in the monitoring period. Clinical characteristics of all participants and participants experiencing hypoglycaemia are shown in Table 1.

Blood glucose The root mean square difference between IG and measurements of blood glucose was $1.6 \pm 0.4 \mathrm{mmol} / 1$ and the absolute relative error was $20 \pm 7 \%$. Glucose concentrations during hypoglycaemia and euglycaemia were $2.8 \pm 0.4$ and $9.0 \pm 1.8 \mathrm{mmol} / \mathrm{l}$, respectively.

ECG variables The changes in measured ECG variables between euglycaemia and hypoglycaemia are summarised in Table 2. QTcB increased from euglycaemia to hypoglycaemia ( $422 \pm 30$ vs $432 \pm 33 \mathrm{~ms} ; p=0.02$ ). QTcF, QTcN and QTcS did not change significantly. During the monitoring period there was a case of prolonged hypoglycaemia with IG $<2.5 \mathrm{mmol} / 1$ for more than $4 \mathrm{~h}$. In this case a QTcS greater than $500 \mathrm{~ms}$ was observed (Fig. 1). Changes in QTcS from euglycaemia to hypoglycaemia were not different between men and women $(9 \pm 20$ vs $8 \pm 8 \mathrm{~ms}$; $p=0.84$ ) or between people with and without neuropathy ( $19 \pm 26$ vs $4 \pm 9 \mathrm{~ms} ; p=0.35$ ).

Heart rate correction The participant-specific correction coefficient, $\alpha$, was $0.20 \pm 0.04$ for the 14 participants with hypoglycaemia. Table 3 shows how the fixed correction formulas compare with QTcS during measurements of hypoglycaemia. QTcB was not significantly different from QTcS $(p=0.08)$, but QTcF $(p=0.02)$ and QTcN $(p=0.04)$ significantly underestimated the QTc during hypoglycaemia. In Fig. 2, the QT/RR intervals during euglycaemia are shown for two participants together with the results of
Table 1 Clinical characteristics of all participants in the study and participants experiencing hypoglycaemia

Data are mean $\pm \mathrm{SD}$ or number (\%) of patients

\begin{tabular}{|c|c|c|}
\hline Variable & $\begin{array}{l}\text { All participants } \\
(n=21)\end{array}$ & $\begin{array}{l}\text { Participants with hypoglycaemia } \\
(n=14)\end{array}$ \\
\hline Sex (male/female) & $13 / 8$ & $9 / 5$ \\
\hline Age (years) & $58 \pm 10$ & $56 \pm 10$ \\
\hline Duration of diabetes (years) & $34 \pm 12$ & $30 \pm 12$ \\
\hline $\mathrm{HbA}_{1 \mathrm{c}}(\%)$ & $7.9 \pm 0.7$ & $7.9 \pm 0.7$ \\
\hline BMI $\left(\mathrm{kg} / \mathrm{m}^{2}\right)$ & $24.8 \pm 2.7$ & $24.4 \pm 1.7$ \\
\hline Insulin (soluble/analogue) & $13 / 8$ & $8 / 6$ \\
\hline Systolic blood pressure (mmHg) & $146 \pm 18$ & $147 \pm 20$ \\
\hline Diastolic blood pressure $(\mathrm{mmHg})$ & $87 \pm 10$ & $90 \pm 9$ \\
\hline \multicolumn{3}{|l|}{ Neuropathy } \\
\hline \multicolumn{3}{|l|}{ Peripheral } \\
\hline Symptomatic & $8(38 \%)$ & $4(29 \%)$ \\
\hline Biothesiometer & $11(52 \%)$ & $7(50 \%)$ \\
\hline \multicolumn{3}{|l|}{ Autonomic } \\
\hline Symptomatic & $5(24 \%)$ & $3(21 \%)$ \\
\hline Beat to beat $<10$ beats $/ \mathrm{min}$ & $8(38 \%)$ & $3(21 \%)$ \\
\hline Smokers & $7(33 \%)$ & $4(29 \%)$ \\
\hline
\end{tabular}


Table 2 Difference in ECG variables between euglycaemia and hypoglycaemia

Data are mean $\pm \operatorname{SD}(n=14)$

\begin{tabular}{lcclll}
\hline Variable & Euglycaemia & Hypoglycaemia & Difference & $95 \%$ CI & $p$ value \\
\hline Heart rate (beats/min) & $73 \pm 12$ & $76 \pm 10$ & 3 & $-2,8$ & 0.22 \\
QT (ms) & $383 \pm 37$ & $385 \pm 41$ & 2 & $-14,18$ & 0.78 \\
QTcB (ms) & $422 \pm 30$ & $432 \pm 33$ & 10 & 2,19 & 0.02 \\
QTcF (ms) & $409 \pm 29$ & $415 \pm 35$ & 6 & $-5,17$ & 0.29 \\
QTcN (ms) & $411 \pm 27$ & $416 \pm 32$ & 6 & $-4,16$ & 0.25 \\
QTcS (ms) & $417 \pm 28$ & $425 \pm 30$ & 9 & $-1,18$ & 0.07 \\
\hline
\end{tabular}

correcting with Bazett's, Fridericia's and the participantspecific correction.

Rate and rhythm disturbances One participant had frequent ventricular ectopic beats but they were present during both euglycaemia and hypoglycaemia. No cases of sinus bradycardia $(<40$ beats/min) or rhythm disturbance were seen.

\section{Discussion}

In this study we confirm previous findings of prolonged QTc corrected by Bazett's formula during spontaneous hypoglycaemia in adults with type 1 diabetes [4, 6] However, Bazett's formula was the only correction method that produced a significantly prolonged QTc from euglycaemia to hypoglycaemia. In comparison with the participantspecific correction formula, we found Bazett's formula to have the lowest mean difference of the three fixed correction formulas. Fridericia's formula and the nomogram method significantly undercorrected the QTc during hypoglycaemia. Only a small, insignificant increase in heart rate was seen during hypoglycaemia, which could indicate either a reduced sympathetic response to hypoglycaemia or simultaneous vagal activation. Nevertheless, it is noteworthy that even an insignificant increase in heart rate results in significant differences among correction formulas. In our study we found no cases of the rate or rhythm disturbances reported by another study on spontaneous hypoglycaemia [4].

The prolongation of QTc observed in the present study is comparable to a previous report of ECG changes during spontaneous hypoglycaemia in an inpatient setting [12]. In outpatient settings using fully automatic measurements of QT and CGM, QTc has been reported to lengthen to a greater degree than seen in this study [4, 13]. However, the use in these studies of fully automatic measurements of QT without manual over-read has been associated with significantly longer QT intervals and measurement errors [15]. Therefore, results obtained using automatic measurements without manual review should be interpreted with caution.

Investigations of changes in QTc during spontaneous hypoglycaemia have only been done using Bazett's formula [4, 12, 13]. Koivikko et al. [20] found that QTc using Bazett's formula was significantly prolonged during clamped hypoglycaemia while Fridericia's formula and the nomogram method resulted in insignificant changes in QTc. The results of our study confirm these findings.

The fact that we observed a case of severely prolonged QTc during hypoglycaemia in one participant while only a modest increase was seen on average could be explained by the concept of the repolarisation reserve articulated by Roden [21]. This concept suggests that a drug or metabolic change such as hypoglycaemia that affects repolarisation
Fig. 1 Measurements of QTcS in euglycaemia (white circles) and hypoglycaemia (black circles) plotted against time of day for participant 1 (male, age 51 years). Also shown is the IG measured by the continuous glucose monitoring (solid line)

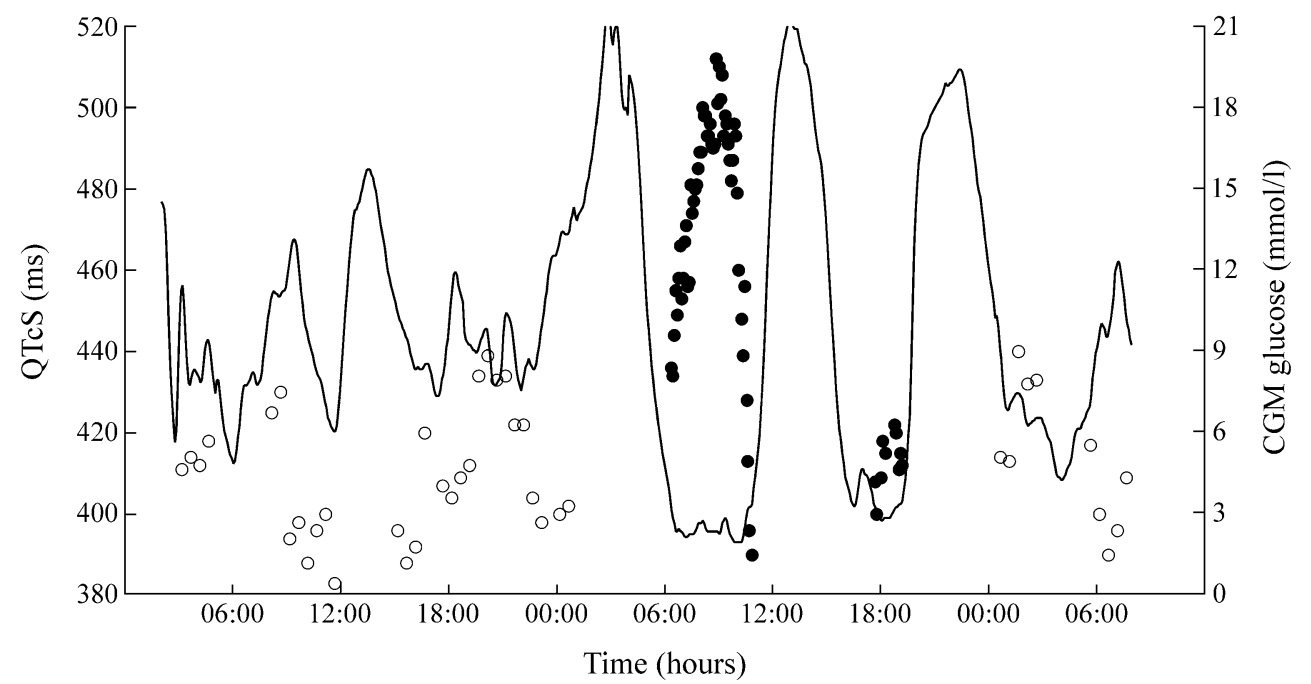


Table 3 Differences during hypoglycaemia between the participantspecific correction formula, QTcS, and the three fixed formulas, $\mathrm{QTcB}, \mathrm{QTcF}$ and QTcN

\begin{tabular}{lccc}
\hline Formula & Difference $(\mathrm{ms})$ & $95 \%$ CI & $p$ value \\
\hline QTcB & $7 \pm 14$ & $-1,15$ & 0.08 \\
QTcF & $-10 \pm 14$ & $-18,-2$ & 0.02 \\
QTcN & $-9 \pm 14$ & $-16,-1$ & 0.04 \\
\hline
\end{tabular}

Data are mean $\pm \mathrm{SD}$

currents may be counteracted by variations in other currents (i.e. the repolarisation reserve), thus maintaining a normal action potential. Reduction in the repolarisation reserve can have a genetic basis or may be acquired and may explain why some individuals show larger prolongations of QTc during hypoglycaemia than others. It is possible that genetic analysis of dead-in-bed patients could reveal mutations responsible for increased susceptibility to QTc prolongation during hypoglycaemia.

We acknowledge that the number of participants developing hypoglycaemia in our study was relatively low. With a larger number of participants it is plausible that all correction formulas would have yielded significant changes in the QTc during hypoglycaemia. In addition, the study population was of greater age and had a longer duration of diabetes than the typical dead-in-bed patient. The sympathoadrenal response to hypoglycaemia causing QT lengthening declines with age and the duration of diabetes. Thus, the participants in this study may have been partly protected against significant QT prolongation, although a reduced adrenalin (epinephrine) response also increases the risk of severe hypoglycaemia. The fact that some participants in this study had diagnosed autonomic

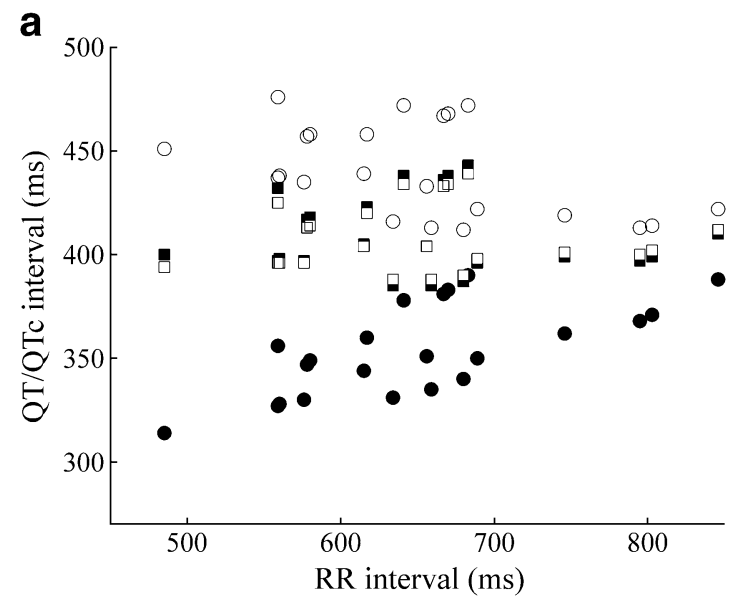

Fig. 2 QT/RR relationship (black circles) for euglycaemic measurements from participant 1 (a) and participant 7 (b). Also shown are QTc/RR relationships using Bazett's method (white circles), Fridericia's method (black squares) and a linear subject-specific method (white squares). In (a), overcorrection by Bazett's formula is seen at low RR intervals (high neuropathy could have affected the results, although such an effect was not observed. It has been hypothesised that autonomic neuropathy could cause increased QTc because of sympathetic overactivity [2]. On the other hand, neuropathy has been shown to attenuate the QTc interval prolongation during hypoglycaemia in type 1 diabetes [14].

Our use of automatic measurements of QT interval on single-lead ECG carries the possibility of inaccuracy, although we sought to minimise this risk by manual review of all measurements. Several factors could have altered the QT and RR measurements used for creating the participantspecific correction of QT. It has been shown that, because of hysteresis in the QT/RR relationship, the QT interval takes approximately $2 \mathrm{~min}$ to adapt to sudden changes in heart rate [22]. Additionally, participants in our study performed everyday activities in which conditions that affect the QT interval could have occurred. The degree to which these factors influenced our results is not known, but the relatively large number of measurements distributed over $72 \mathrm{~h}$ is expected to have minimised their effect. A circadian rhythm of QTc has been reported using fixed heart rate correction formulas, but it has been suggested that this merely reflects the circadian pattern of heart rate [11]. If a circadian pattern of QTc exists it may have affected our results, since it was not considered in our analysis.

The use of a CGM system could have resulted in false negative or false positive measurements of hypoglycaemia in our study. Bode et al. [23] tested the accuracy of the CGM system used in our study by pairing home blood glucose meter readings with CGM meter readings. The relative error between $\mathrm{CGM}$ and blood glucose measurements of $21.3 \%$ corresponds well with the $20.7 \%$ found in our study. The specificity of the CGM system for detecting

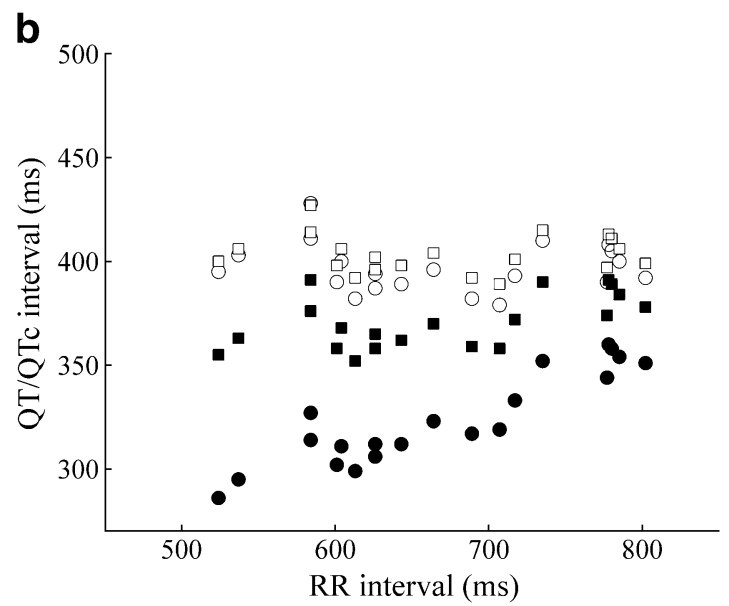

heart rate), while Fridericia's formula is closer to the subject-specific method. The opposite is seen on in (b), where Fridericia's formula undercorrects the QTc, while Bazett's formula is closer to the subjectspecific method 
hypoglycaemia (threshold, $3.9 \mathrm{mmol} / \mathrm{l}$ ) is $90 \%$ [23], indicating that fewer than $10 \%$ of hypoglycaemia measurements are false positives. Using our threshold of $>5 \mathrm{mmol} / 1$ for euglycaemia, the corresponding sensitivity of the CGM system is $91 \%$ [23], indicating that fewer than $9 \%$ of euglycaemia measurements are false negatives. These numbers show that the estimated number of misclassified CGM measurements in the present study was fewer than $10 \%$ of all measurements.

It is clearly important to clarify the possible proarrhythmic effect of hypoglycaemia and its role in relation to the dead-in-bed syndrome. Although hypoglycaemia in general was only associated with a modest prolongation of QTc, we observed a case of prolonged severe hypoglycaemia in which the QTc exceeded $500 \mathrm{~ms}$. Larger trials investigating how QTc is altered by spontaneous hypoglycaemia are needed but should consider the effect of the heart rate correction formula as they may bias the results in either direction. If possible, participant-specific heart rate correction of QT should be considered in order to achieve QTc uncorrelated with heart rate.

Acknowledgements This study was funded by Novo Nordisk.

Duality of interest The authors declare that there is no duality of interest associated with this manuscript. Several authors are employees of Novo Nordisk or Steno Diabetes Center, which is owned by Novo Nordisk. Novo Nordisk funded the study but there are no financial interests for the company in the publication of this manuscript.

\section{References}

1. Tattersall RB, Gill GV (1991) Unexplained deaths of type 1 diabetic patients. Diabet Med 8:49-58

2. Weston PJ, Gill GV (1999) Is undetected autonomic dysfunction responsible for sudden death in type 1 diabetes mellitus? The 'dead in bed' syndrome revisited. Diabet Med 16:626-631

3. Collier A, Matthews DM, Young RJ, Clarke BF (1987) Transient atrial fibrillation precipitated by hypoglycaemia: two case reports. Postgrad Med J 63:895-897

4. Gill GV, Woodward A, Casson IF, Weston PJ (2009) Cardiac arrhythmia and nocturnal hypoglycaemia in type 1 diabetes - the 'dead in bed' syndrome revisited. Diabetologia 52:42-45

5. Marques JLB, George E, Peacey SR et al (1997) Altered ventricular repolarization during hypoglycaemia in patients with diabetes. Diabet Med 14:648-654
6. Robinson RT, Harris ND, Ireland RH, Macdonald IA, Heller SR (2004) Changes in cardiac repolarization during clinical episodes of nocturnal hypoglycaemia in adults with type 1 diabetes. Diabetologia 47:312-315

7. Yap YG, Camm AJ (2003) Drug induced QT prolongation and torsades de pointes. Heart 89:1363-1372

8. Straus SMJM, Kors JA, de Bruin ML et al (2006) Prolonged QTc interval and risk of sudden cardiac death in a population of older adults. J Am Coll Cardiol 47:362-367

9. Bazett HC (1920) An analysis of the time relations of electrocardiograms. Heart 7:353-370

10. Funck-Brentano C, Jaillon P (1993) Rate-corrected QT interval: techniques and limitations. Am J Cardiol 72:17B-22B

11. Malik M, Farbom P, Batchvarov V, Hnatkova K, Camm AJ (2002) Relation between QT and RR intervals is highly individual among healthy subjects: implications for heart rate correction of the QT interval. Heart 87:220-228

12. Murphy NP, Ford-Adams ME, Ong KK et al (2004) Prolonged cardiac repolarisation during spontaneous nocturnal hypoglycaemia in children and adolescents with type 1 diabetes. Diabetologia 47: 1940-1947

13. Suys B, Heuten S, de Wolf D et al (2006) Glycemia and corrected QT interval prolongation in young type 1 diabetic patients. What is the relation? Diab Care 29:427-429

14. Lee SP, Yeoh L, Harris ND et al (2004) Influence of autonomic neuropathy on QTc interval lengthening during hypoglycemia in type 1 diabetes. Diabetes 53:1535-1542

15. Molnar J, Ranade V, Cvetanovic I, Molnar Z, Somberg JC (2006) Evaluation of a 12-lead digital Holter system for 24-hour QT interval assessment. Cardiology 106:224-232

16. O'Brien IA, O'Hare P, Corrall RJ (1986) Heart rate variability in healthy subjects: effect of age and the derivation of normal ranges for tests of autonomic function. Br Heart J 55:348-354

17. McLaughlin NB, Campbell RW, Murray A (1995) Comparison of automatic QT measurement techniques in the normal 12 lead electrocardiogram. Br Heart J 74:84-89

18. Fridericia LS (1920) Die Systolendauer im Electrokardiogramm bei normalen Menschen und bei Herzkranken. Acta Med Scand 53:469-486 (article in German)

19. Karjalainen J, Viitasalo M, Manttari M, Manninen V (1994) Relation between QT intervals and heart rates from 40 to 120 beats/min in rest electrocardiograms of men and a simple method to adjust QT interval values. J Am Coll Cardiol 23:1547-1553

20. Koivikko ML, Karsikas M, Salmela PI et al (2008) Effects of controlled hypoglycaemia on cardiac repolarisation in patients with type 1 diabetes. Diabetologia 51:426-435

21. Roden DM (2008) Repolarization reserve: a moving target. Circulation 118:981

22. Lau CP, Freedman AR, Fleming S, Malik M, Camm AJ, Ward DE (1988) Hysteresis of the ventricular paced QT interval in response to abrupt changes in pacing rate. Cardiovasc Res 22:67-72

23. Bode B, Gross K, Rikalo N et al (2004) Alarms based on real-time sensor glucose values alert patients to hypo- and hyperglycemia: the guardian continuous monitoring system. Diabetes Technol Ther 6:105-113 